\section{Olbers' paradox} in concert halls?

SIR - There are some ways of looking at problems in physics that transcend the fields in which they originated. An important case is statistical mechanics, developed in the latter half of the nineteenth century for the kinetic theory of gases.

But traps await those who do not comprehend the limitations of the original theory. The kinetic theory cannot apply to systems the size of the meanfree-path between collisions of molecules, $10^{-7} \mathrm{~m}$ at atmospheric pressure, or $10^{-1} \mathrm{~m}$ in a good vacuum, for collisions with the boundaries then predominate.

In about 1900, W. C. Sabine attempted a similar theory of room acoustics, calculating a 'mean-free-path' of fronts of sound between their encounters with the boundaries of rooms. This path was of the order of the size of the room; its exact length was the subject of controversy for 75 years, for the mathematics are formidable. There is no unique and singular path length, as W. B. Joyce showed in $1975^{1}$.

Recently, a stronger reason for abandoning Sabine's model has been found. The presence of even moderate amounts of sound-absorbing materials in a room makes impossible the uniform field of energy, slowly varying with time, that Sabine supposed ${ }^{2}$. The audience in a concert hall (or any auditorium) is highly absorbing, so the existence of such a field is out of the question. The physics of concert halls is the physics of flows of energy and information from the orchestra to the audience. The correct theory is based on physical acoustics and the encoding of information in transient structures of waves: the physics pioneered by Denis Gabor ${ }^{3}$.

What has this to do with Olbers' paradox, the question of why the sky is dark at night? If the stars were eternal and the Universe infinite, space would be filled with a uniform luminosity, for every line of sight would intercept the surface of a star. This problem has engaged astronomers for 400 years. The solution accepted now is that the time required for a uniform field to develop is much longer that the lifetime of galaxies. It is not necessary to invoke relativity or an expanding Universe ${ }^{4}$.

The existence of strong absorption in

\section{Correction}

The first sentence of the letter from N.W. Pirie (Nature 351, 704; 1991) should read: "By using the title "Sperm warfare", Daedalus' research team emphasizes that it thinks only of aggression as a factor in sperm competition." The incorrect Daedalus title was an editorial error. $\square$ the Universe is not so clear as in concert halls. Its presence makes the 'timeconstant' so short that too few reflections occur to generate a uniform field; the number of these reflections is inversely proportional to the strength of the absorption. Surprisingly, neither scale nor speed of propagation makes a difference; the same relation obtains for molecules in a microvacuum, sound in a concert hall or light in the Universe.

In this sense, a time-varying classical Universe is like a time-varying classical concert hall. The mediaeval idea that music and astronomy are closely related is true in this case. Although Sabine's theory clearly is wrong, he instituted another paradox by using it to design the world's finest concert hall, Boston Symphony Hall. I speculate that he drew on the earlier, correct approach to the problem by Joseph Henry 5 .

For nearly 100 years we have suffered concert halls designed to a wrong theory. No designer since Sabine has mastered the art, so the thousands of auditoria built each year are, at best, mediocre. A good theory will have a great practical effect.

JAMES B. LEE

Department of Mechanical Engineering, Portland State University,

Portland, Oregon 97207-0751, USA

1. Joyce, W. B. J. Acoust. Soc. Am. 58, 643-655 (1975) 2. Lee, J. B. J. Acoust. Soc. Am. 85, 2680-2681 (1989) 3. Gabor, D. in NATO Symp. Opt. Acoust. Holog. (Plenum, New York, 1972)

Wesson, P. S. Astrophys. J. 367, 399-406 (1991) 5. Henry, J. Proc. Am. Assoc. 10, 119-135 (1856).

\section{Object awareness}

SIR - Libet ${ }^{1}$ makes three comments about our work. (1) We do not dispute that some forced-choice perceptual tests can be performed successfully without awareness ${ }^{2,3}$, but this is irrelevant to our recent claim $^{4}$. We described tests of visual perception of orientation, shape and size which our brain-damaged patient (D.F.) failed; she failed them whether we asked her for verbal reports, or for manual pantomime judgements, or for forced-choice judgements. In short, on all the perceptual tests we used, there was no evidence for awareness of these object qualities. By contrast, when we asked her to act on the objects, she did so with great success and with apparently normal skill. It was this remarkable dissociation between D.F.'s perceptual judgements and her visuomotor control that led us to postulate a separation in the underlying neural architecture.

(2) Libet then argues that such a separation of brain systems for perception and action need not imply a general separation between conscious and unconscious functions. Again this is uncontentious. We were making the much more specific suggestion that D.F.'s profound visual-form agnosia ${ }^{5}$ might be caused by the destruction or disconnection of perceptual systems that normally mediate conscious visual-object perception; and that her preserved visual skills are probably exploiting relatively intact structures which in the normal individual mediate the visuomotor control of prehension and other motor acts.

Of course, unlike our patient, normal subjects can have conscious 'perceptual' information about the visual objects and targets which they grasp or foveate; but we believe that the processing mediating that experience is distinct from the visuomotor computations that modulate the movements themselves. In fact, just like D.F., normal subjects may generate visually guided eye and limb movements on the basis of covert visual information that is inaccessible to consciousness ${ }^{6}$. Indeed, we suspect that an important feature of conscious visual perception is that it can remain refractory to the visual information to which visuomotor networks are often explicitly sensitive.

(3) Finally, we do not deny that in some cases information processing might become conscious or unconscious simply as a function of the duration of relevant afferent neural activity. But such a conclusion is by no means forced on us by Libet's experimental data. The fact that a single thalamic pathway was electrically stimulated in his two experiemental conditions (long versus short duration ) does not ensure that one and the same perceptual system was activated in the two conditions of stimulation. Furthermore, the distinction between brief and longer-lasting activation cannot account for the dissociations we observed in D.F.; there is no plausible reason to suppose that visual pathways were activated more briefly in one type of task than in the other. Indeed, the objects whose qualities she failed to perceive, but was able to act on, were always present for at least several seconds, and sometimes for up to a minute.

M. A. GOODALE

A. D. MILNER

L. S. JAKOBSON

D. P. CAREY

Psychological Laboratory,

University of St Andrews,

St Andrews, Fife KY16 9J, UK

1. Libet, B. Nature 351, 194-195 (1991)

2. Meeres, S. L. \& Graves, R. E. Neuropsychologia 28. 1231-1237 (1990)

3. Cowey, A. in The Neuropsychology of Consciousness (eds Milner, A. D. Rugg, M. D.). (Academic, London, in the press)

4. Goodale, M. A., Milner, A. D., Jakobson L. S. \& Carey, D. P. Nature 349, 154-156 (1991)

5. Milner, A. D. et al. Brain 114, 405-428 (1991)

6. Goodale, M. A., Pelisson, D. \& Prablanc, C. Nature 320. 748-750 (1986) 